\title{
Effects of melatonin and SkQ1 long-term treatment during aging and development AMD-like retinopathy
}

\author{
Darya $V$. Telegina \\ ICG SB RAS, Novosibirsk, Russia \\ telegina@bionet.nsc.ru
}

\author{
Oyuna S. Kozhevnikova \\ ICG SB RAS, Novosibirsk, Russia \\ oidopova@bionet.nsc.ru
}

\author{
Anzhela Z. Fursova \\ ICG SB RAS, Novosibirsk, Russia \\ anzhellafursova@yandex.ru
}

\begin{abstract}
Melatonin and antioxidant SkQ1 act like mitochondria-targeted antioxidants, which concentrate in mitochondria at relatively high levels and they may prevent mitochondrial damage during retinal aging and development of age-related retinal disease such as age-related macular degeneration (AMD). However, detailed effects of melatonin and SkQ1 on the biochemical mechanisms underlying therapeutic effect of these drugs during retinal aging and AMD progression remain unclear. Using Wistar rats with normal aging process and senescence-accelerated OXYS rats, which spontaneously develop a phenotype similar to human age-related disorders including AMD-like retinopathy, we found that treatment of SkQ1 and melatonin decreased the incidence and severity of retinopathy in OXYS rats. In Wistar rats, which do not naturally develop retinopathy, ophthalmoscopic inspections did not reveal pathological alterations in the retina of melatonin and SkQ1treated rats. SkQ1 decreased p62/SQSTM1 protein but not mRNA levels in both OXYS and Wistar rat's retinas as compared of control rats. We observed reduced level of VDAC1 and increased level of glutaminase by long-term treatment of melatonin and SkQ1 in retina of Wistar rats but not OXYS rats. Taken together, our data indicated that long-term treatment of melatonin and mitochondria-targeted antioxidant SkQ1 may retard an agerelated decline in the adaptability of retinal cells and may be considered as a strategy to slow down AMD. At the same time effects of melatonin and SkQ1 on molecular events may be different depending on genotype and disease.
\end{abstract}

Keywords - melatonin, SkQ1, aging, retina, AMD, OXYS rats, Wistar rats

\section{Introduction}

Today the percent of older people are on a dramatic increase result in greater elderly human with age-related diseases such as age-related macular degeneration (AMD). AMD is the predominant cause of visual loss in the macula in old people and is characterized by degeneration of retinal pigment epithelium and photoreceptors, impaired autophagy, DNA damage, mitochondrial dysfunction, increased levels of ROS and impaired of blood vessels. It is important that AMD is characterized by retina alterations similar to normal retinal aging. This fact complicates the study of AMD pathogenesis and the search for new therapy drugs.

Accumulating evidence indicates that the melatonin, Nacetyl-5-methoxytryptamine, and mitochondria-targeted antioxidant SkQ1 have a positive effects on AMD treatment [1]. Both SkQ1 and melatonin act like mitochondria-targeted antioxidants, which concentrate in mitochondria at relatively high levels and they may prevent mitochondrial damage in AMD. Melatonin scavenged free radicals generated in the mitochondria, to reduce electron leakage from the respiratory complexes and to improve ATP synthesis, maintains reduced glutathione levels within the mitochondria thereby enhancing the antioxidative potential. Moreover, melatonin was shown to influence autophagy, to stimulate the activity of telomerase, restoration of inner blood-retina barrier integrity, reduced VEGF and NO levels in the aged retina [2]. SkQ1 (10-(6plastoquinonyl) decyltriphenyl-phosphonium) is plastoquinol derivative modified by a lipophilic cation then accumulate in the mitochondrial matrix and modulate of mitochondrial superoxide formation at specific sites of Complex I and III [3]. There are evidence that SkQ1 to slow down aging and to retard, arrest, and in some cases even reverse the development of many age-related eye diseases [4]. However, detailed effects of melatonin and SkQ1 on the biochemical mechanisms underlying therapeutic effect of these drug during retinal aging and AMD progression remain unclear.

Here, we analyzed the effects of melatonin and SkQ1 longterm treatment during aging and development AMD using by animal model. We used Wistar rats with normal aging process and senescence-accelerated OXYS rats, which spontaneously develop a phenotype similar to human age-related disorders including AMD-like retinopathy. The clinical signs of AMDlike retinopathy appear by the age of 3 months. Significant pathological changes detected at the age of 12 months and manifest themselves as excessive accumulation of lipofuscin and amyloid in the RPE regions, disturbances in the morphology of the RPE sheet, including an increase in the proportion of multinucleated cells, hypertrophy, distortion of cell shape, and reactive gliosis [5]. We explored the in vivo effects of melatonin and SkQ1 long-term treatment (6 months) in the retina of Wistar and OXYS rats during aging and AMD progression on the expression of autophagy-associated genes ( $\mathrm{Nbr}$, Atg7, Becn 1, Gabarapl1, LC3B, p62/SOSTM1), mitochondria-associated genes (VDAC1 and mtTFA) and glutaminase that generates glutamate from glutamine.

\section{Methods}

To comparison of the effects of melatonin and SkQ1 longterm treatment on aged retina, 12-month-old OXYS and Wistar rats were randomly assigned to one of three groups $(n=10)$. The first group consumed a control diet, the second group supplemented with $0.04 \mathrm{mg}$ of melatonin (Melaxen; Unipharm, New York, NY, USA) per $\mathrm{kg}$ of body and the third group supplemented with SkQ1 $250 \mathrm{nmol}$ per $\mathrm{kg}$ of body per day from the age of 12 to 18 months. The animals were provided with standard rodent feed and water ad libitum. SkQ1 was production by Institute of Mitoengineering of Moscow State University (Moscow, Russia).

We used standard techniques of Western-blot and qPCR to estimate effects of melatonin and SkQ1 on retina [5]. All the rats were examined by an ophthalmologist two times: before supplementation at the age of 12 months, and during the treatment at ages 18 months as decribed in [6]. Five days after the last eye examination, the rats were euthanized by $\mathrm{CO}_{2}$ asphyxiation and decapitated. All animal procedures were 
in compliance with the Association for Research in Vision and Ophthalmology statement for the Use of Animals in Ophthalmic and Vision Research and the European Communities Council Directive 86/609/EES.

\section{Results}

Here, we observed similar effects of melatonin and SkQ1 in the retina of Wistar rats. Preliminary examination showed that there was no difference between 12-month-old OXYS rats assigned to experimental and control groups. By the age of 22 months, retinopathy in control OXYS rats progressed. Both SkQ1 and melatonin decreased the incidence and severity of retinopathy in OXYS rats. In Wistar rats, which do not naturally develop retinopathy, repeated inspection did not reveal pathological alterations in the retina of melatonin and SkQ1treated rats.

Protein level of VDAC1 was greater in retina of Wistar rats as compared OXYS rats. Treatment of melatonin and SkQ1 decreased VDAC1 level in Wistar rats and no influence on OXYS rats. VDAC1, also known as mitochondrial porin, associate with NADH oxidation and thus plays a role in cellular redox mechanisms. There are evidence that VDAC1 increases with age and may have negative effects by interrupting mitochondrial pore opening and closure. Also VDAC1 may interacts with $\mathrm{A} \beta$, and phosphorylated tau may in turn block mitochondrial pores, leading to mitochondrial dysfunction [7]. Based on current study observations, we propose that reduced levels of VDAC1 by long-term treatment of melatonin and SkQ1 leading to normal mitochondrial function in Wistar rats.

The protein level of glutaminase was lower in Wistar rats as compared OXYS rats. Treatment of melatonin and SkQ1 increased protein level of glutaminase in Wistar rat's retina. In mammalian cells, glutamine is converted to glutamate by glutaminase (GLS), which functions as the rate-limiting enzyme, and then to $\alpha$-ketoglutarate $(\alpha-K G) . \alpha-K G$ enters the tricarboxylic acid cycle, not only for the generation of ATP via oxidative phosphorylation, but also for the production of acetylcoA as a critical precursor for the synthesis of lipids and nucleotides [8]. On other hand, glutamate is the main excitatory neurotransmitter in the retina, but it is neurotoxic when present in excessive amounts. On the basis of these results, it might be presumed that rat retinal glutaminase level is regulated by antioxidants.

We observed that treatment of SkQ1 dramatically decreased p62/SQSTM1 protein but not mRNA levels in both OXYS and Wistar rats as compared of control rats. p62/SQSTM1 is a scaffold protein for many signaling pathways, such as autophagy, apoptosis and inflammatory. Analyze of mRNA level of autophagy-dependent genes ( $\mathrm{Nbr}, \operatorname{Atg} 7$, Becn 1 , Gabarapl1, LC3B) demonstrated increasing mRNA level of Beclin 1 in OXYS rats after SkQ1 and melatonin treatment. The
Beclin1 protein involved in initiation of autophagy. The treatment of SkQ1 is not change the protein level of Atg7 in the retina of OXYS and Wistar rats.

Summary, our data indicated that long-term treatment of melatonin and mitochondria-targeted antioxidant SkQ1 may be the different effects on molecular events resulting from different in genotype and environment.

\section{Conclusion}

Thus, using Wistar rats with normal aging process and senescence-accelerated OXYS rats, which spontaneously develop AMD-like retinopathy, we demonstrated that long-term treatment of melatonin and mitochondria-targeted antioxidant SkQ1 may retard an age-related decline in the adaptability of retinal cells and may be considered as a strategy to slow down AMD.

\section{ACKNOWLEDGMENT}

This study was supported by the Russian Foundation for Basic Research (18-015-00320) and by a Russian Scientific Foundation Grant (19-15-00044).

\section{REFERENCES}

[1] Blasiak, J., Reiter, R. J., Kaarniranta, K. (2016). Melatonin in retinal physiology and pathology: the case of age-related macular degeneration. Oxidative medicine and cellular longevity, 2016. https://doi.org/10.1155/2016/6819736

[2] Rastmanesh, R. (2011). Potential of melatonin to treat or prevent agerelated macular degeneration through stimulation of telomerase activity. Medical hypotheses, 76(1), 79-85. https://doi.org/10.1016/j.mehy.2010.08.036

[3] Ježek, J., Engstová, H., \& Ježek, P. (2017). Antioxidant mechanism of mitochondria-targeted plastoquinone SkQ1 is suppressed in aglycemic HepG2 cells dependent on oxidative phosphorylation. Biochimica et Biophysica Acta (BBA)-Bioenergetics, 1858(9), 750-762. https://doi.org/10.1016/j.bbabio.2017.05.005

[4] Anisimov V. N. et al. (2011) Effects of the mitochondria-targeted antioxidant SkQ1 on lifespan of rodents. Aging (Albany NY), 3(11), 1110. https://doi.org/10.18632/aging.100404

[5] Kozhevnikova, O. S., Telegina, D. V., Tyumentsev, M. A., \& Kolosova, N. G. (2019). Disruptions of Autophagy in the Rat Retina with Age During the Development of Age-Related-Macular-Degeneration-like Retinopathy. International Journal of Molecular Sciences, 20(19), 4804. https://doi.org/10.3390/ijms20194804

[6] Kolosova, N. G., et al. (2018). p62/SQSTM1 coding plasmid prevents age related macular degeneration in a rat model. Aging (Albany NY), 10(8), 2136.

[7] Manczak, M., Reddy, P. H. (2012). Abnormal interaction of VDAC1 with amyloid beta and phosphorylated tau causes mitochondrial dysfunction in Alzheimer's disease. Human molecular genetics, 21(23), 5131-5146. https://doi.org/10.1093/hmg/dds360

[8] Cai, W. F., Zhang, C., Wu, Y. Q., Zhuang, G., Ye, Z., Zhang, C. S., Lin, S. C. (2018). Glutaminase GLS1 senses glutamine availability in a nonenzymatic manner triggering mitochondrial fusion. Cell research, 28(8), 865-867. https://doi.org/10.1038/s41422-018-0057-z 